\title{
Micobiota do Queijo Artesanal Serrano produzido em Santa Catarina, Brasil
}

\author{
Mycobiota of Serrano Artisanal Cheese produced in Santa Catarina, Brazil
}

\author{
Marcella Nunes Pereira*, Juliana Regina da Silva, Ítala Sabrina Fernandes Freire, Lívia Colletti \\ Escatolin e Stéfano Caon Tallamini
}

Universidade Federal de Santa Catarina, Florianópolis, SC, Brasil. *Autor para correspondência: marcella.mnp@gmail.com.

Submissão: 04/07/2018 / Aceite: 01/07/2019

\begin{abstract}
RESUMO
O Queijo Artesanal Serrano é um produto nativo do sul do Brasil, produzido por mão de obra familiar a partir do leite cru, e que vem buscando a obtenção da sua indicação geográfica. Muitas das características que particularizam os tipos de queijo são dadas pela sua micobiota natural. $\mathrm{O}$ objetivo deste estudo foi determinar as espécies fúngicas presentes em 20 amostras de Queijo Artesanal Serrano de quatro períodos de maturação (14, 21, 28 e 35 dias), produzidos em outubro de 2017, em Santa Catarina. A identificação dos bolores foi feita por chaves de identificação e das leveduras por MALDI-TOF. Os bolores foram isolados em 28 das 80 amostras e com espécies variáveis, sendo algumas contaminantes. Por outro lado, as leveduras foram detectadas em todas as amostras, com alguns gêneros mais frequentes, principalmente o Kluyveromyces lactis, presente em 27 amostras, e algumas espécies de Candida spp. As espécies identificadas já foram isoladas em outros queijos artesanais brasileiros, produzido em locais com características de clima e relevo semelhantes ao do Queijo Artesanal Serrano.
\end{abstract}

PALAVRAS-CHAVE: bolores, fungos, identificação, leveduras, micobiota natural.

\begin{abstract}
Serrano Artisanal Cheese is a native product from the South region of Brazil, produced by family labor from raw milk, which seeks to obtain its geographical indication. Many of the characteristics that particularize the types of cheese are given by its natural mycobiota. The objective of this study was to determine the fungal species present in 20 samples of Serrano Artisanal Cheese from four ripening periods $(14,21,28$, and 35 days), produced in October 2017, in Santa Catarina. Identification of molds was made by identification keys and yeasts by MALDI-TOF. The molds were isolated in 28 of the 80 samples and with various species, some being attributed to contamination. On the other hand, yeasts were detected in all samples, with some genera being more frequent, specially Kluyveromyces lactis, present in 27 samples, and some species of Candida spp. The identified species have already been isolated in other Brazilian artisanal cheeses produced in places with similar climate and relief to that of Serrano Artisanal Cheese.
\end{abstract}

KEYWORDS: moulds, fungal, identification, yeasts, natural micobiota.

O Queijo Artesanal Serrano (QAS) é um produto típico do sul do Brasil, com mais de duzentos anos de tradição, característico de uma região de $16.000 \mathrm{~km}^{2}$ entre os Campos de Cima da Serra no nordeste do Rio Grande do Sul (RS) e o Planalto Sul de Santa Catarina (SC) (CÓRDOVA et al. 2010, CÓRDOVA et al. 2011). Sua fabricação é feita por mão de obra familiar, com o leite bovino cru obtido na propriedade. A forma como este queijo é fabricado, os fatores climáticos e do ambiente de produção afetam a composição de sua micobiota, que torna este produto particular e característico da região produtora (CÓRDOVA et al. 2011, PEREIRA et al. 2014). Alguns dos componentes que mais alteram as caraterísticas físico-químicas e sensoriais dos queijos são os bolores e leveduras (JODRAL et al. 1993). Estes microrganismos estão naturalmente presentes no leite cru e, consequentemente, no produto desta matéria-prima. Sua ocorrência ainda pode ser atribuída à contaminação pelos utensílios utilizados ou pelo ambiente de produção (JAY 2005).

O QAS Catarinense vem se destacando nos últimos anos, ganhando em 2016 uma regulamentação 
própria, a lei $n^{\circ} 17.003$, para o controle da sua produção e comercialização (SANTA CATARINA 2016). Levando em consideração a importância para o sustento da agricultura familiar da região produtora e toda a história e cultura deste queijo, diversos estudos para a caracterização deste produto estão sendo realizados para que se obtenha a sua indicação geográfica. Este trabalho tem como objetivo determinar a micobiota presente no QAS, que auxilia na construção do seu sabor, textura e aparência.

\section{Materiais e Reagentes}

Ácido trifluoroacético (Merck, Darmstadt, Hesse, Alemanha), matriz HCCA (Sigma-Aldrich, St. Louis, MO, EUA), ágar batata dextrose (PDA), ágar extrato de levedura Czapek (CYA), ágar extrato de malte (MEA), ágar glicerol nitrato 25\% (G25N) e água peptonada tamponada (Himedia, Mumbaí, Maharashtra, Índia), cloranfenicol (Vetec, Rio de Janeiro, RJ, Brasil), corante azul de algodão (NewProv, Curitiba, PR, Brasil) e placas de Petri (Kasvi, Curitiba, PR, Brasil). Os equipamentos consistem em: agitador de tubos (Marconi, São Paulo, SP, Brasil), autoclave e contador de colônias (Phoenix®), balança analítica (Shimadzu, Kioto, Japão), capela de fluxo contínuo (Veco, Campinas, SP, Brasil), estereoscópio e microscópio (Carl Zeiss, Jena, Turíngia, Alemanha), estufa bacteriológica (Fanen, São Paulo, SP, Brasil), MALDI-TOF Biotyper 4.0 (BrukerDaltonics, Bremen, Alemanha) e espectrômetro de massa Autoflex III (BrukerDaltonik, Billerica, MA, EUA).

\section{Coleta e preparação das amostras}

Técnicos extensionistas da Empresa de Pesquisa Agropecuária e Extensão Rural de Santa Catarina (EPAGRI), que já trabalham diretamente assistindo os produtores, realizaram as coletas. Estas ocorreram nas 20 propriedades produtoras pertencentes à Associação de Produtores de Queijo Artesanal Serrano da Serra Catarinense dos municípios de Lages e São Joaquim, SC. As amostras foram acondicionadas em papel craft e sacos plásticos estéreis e enviadas ao Laboratório de Micotoxinas e Contaminantes Alimentares (LABMICO), da Universidade Federal de Santa Catarina (UFSC) em caixas isotérmicas com gelo reutilizável.

Foram analisadas 80 amostras do Queijo Artesanal Serrano produzidos em setembro de 2017, sendo 20 de cada período de maturação (14, 21, 28 e 35 dias).

\section{Preparo das amostras}

Foram pesadas $25 \mathrm{~g}$ de cada amostra, em duplicata, de maneira asséptica, adicionados $225 \mathrm{~mL}$ de água peptonada tamponada $(0,1 \%)$ e agitados por um minuto no agitador automático. Posteriormente, foram realizadas diluições $\left(10^{-1}\right.$ a $\left.10^{-6}\right)$ e plaqueadas por espalhamento em superfície em ágar batata dextrose (PDA). As placas foram mantidas em estufa a $25{ }^{\circ} \mathrm{C}$ por cinco dias e, após este período, as diferentes colônias foram contadas em unidades formadoras de colônia (log UFC g ${ }^{-1}$ ) (SILVA et al. 2010).

\section{Identificação de bolores}

A identificação dos bolores foi realizada conforme a metodologia e chaves de identificação de PITT \& HOCKING (2009). As colônias que apresentaram morfologias diferentes na contagem fúngica foram isoladas em PDA por cinco dias e repicadas nos meios de cultura diferenciais: ágar extrato de malte, ágar czapek extrato de levedura e ágar glicerol nitrato a $25 \%$, e submetidas ao microcultivo. Todas as placas foram mantidas novamente em estufa a $25{ }^{\circ} \mathrm{C}$ por cinco dias, e suas características confrontadas com a chaves de identificação, chegando aos seus respectivos gêneros e espécies.

\section{Identificação de leveduras}

A fim de identificar as leveduras, as diferentes colônias também foram repicadas para isolamento em PDA e mantidas em estufa a $25^{\circ} \mathrm{C}$ por dois dias. Posteriormente foram enviadas sob refrigeração para 0 Instituto de Ciências Básicas da Saúde (ICBS) da Universidade Federal do Rio Grande do Sul (UFRGS), onde foram realizadas as identificações via espectrometria de massas por ionização e dessorção a laser assistida por matriz e detecção do tempo de voo (MALDI-TOF). Para tanto, aproximadamente $50 \mu \mathrm{g}$ de cada colônia foram colocados sobre uma placa de aço inox, recobertos por uma matriz HCCA (ácido $\alpha$ ciano-4 hidroxicinâmico) e secos a temperatura ambiente $\left(20^{\circ} \mathrm{C}\right)$. Os espectros de massa foram coletados nas faixas de massa entre 2.000-20.000 m/z e analisados usando o software MALDI Biotyper 4.0 (Bruker Daltonik, Bremen, Alemanha) para o cruzamento com os dados da biblioteca de padrões do programa. A similaridade de cada espectro foi calculada resultando em logaritmos que foram de zero (sem homologia) a 3,0 ( $100 \%$ de homologia). As pontuações $\geq 1,7$ foram consideradas identificações de gênero confiáveis, e as $\geq 2,0$ identificações de gênero e espécie confiáveis (NAGY et al. 2009).

\section{Análise estatística}

As médias das contagens foram submetidas à Análise de Variância (ANOVA) no programa estatístico Statistica 13.3. As médias significativamente diferentes $(p>0,05)$, foram comparadas pelo teste de Tukey a $5 \%$, sendo confrontadas as variações ao longo dos períodos de maturação. A identificação dos bolores e 
leveduras componentes do QAS foram analisadas por estatística descritiva e sensoriais, como sabor, odor e textura. Por outro lado, espécies patogênicas também podem estar presentes. A composição da micobiota presente no queijo é de fundamental importância para a produção de suas características, comprometendo a qualidade e a seguridade do produto. No QAS produzido no mês de setembro de 2017, submetido a quatro diferentes períodos de maturação, foram encontradas as espécies de fungos filamentosos descritas na Tabela 1.

Tabela 1. Espécies de fungos filamentosos presentes no Queijo Artesanal Serrano ao longo dos períodos de maturação.

Table 1. Species of filamentous fungi present in Serrano Artisanal Cheese during ripening periods.

\begin{tabular}{|c|c|c|c|c|c|}
\hline \multirow{2}{*}{ Gêneros } & \multirow{2}{*}{ Espécies } & \multicolumn{4}{|c|}{ Frequência de amostras com presença dos bolores } \\
\hline & & 14 dias* & 21 dias* & 28 dias* & 35 dias* \\
\hline Aspergillus & candidus & $2(10 \%)$ & - & - & - \\
\hline Byssochlamys & nivea & $2(10 \%)$ & $2(10 \%)$ & $3(15 \%)$ & - \\
\hline Cladosporium & herbarum & $1(5 \%)$ & $1(5 \%)$ & - & - \\
\hline \multirow{2}{*}{ Fusarium } & oxysporum & - & $2(10 \%)$ & $1(5 \%)$ & - \\
\hline & solani & - & - & $1(5 \%)$ & - \\
\hline Geotrichum & candidum & $2(10 \%)$ & $4(20 \%)$ & - & - \\
\hline Mucor & hiemalis & - & $2(10 \%)$ & - & - \\
\hline \multirow{3}{*}{ Penicillium } & chrysogenum & $1(5 \%)$ & $1(5 \%)$ & - & - \\
\hline & commune & - & - & $2(10 \%)$ & - \\
\hline & expansum & - & - & $1(5 \%)$ & - \\
\hline Sem crescimento & & $12(60 \%)$ & $8(40 \%)$ & $12(60 \%)$ & $20(100 \%)$ \\
\hline
\end{tabular}

O crescimento de fungos filamentosos foi relativamente baixo, variando entre $40 \%$ e $100 \%$ das amostras do período em análise sem o crescimento de nenhuma espécie. Tal fato já era esperado, devido a este queijo não ter a característica de possuir bolores aparentes. Dentre as espécies encontradas estão o $A$. candidus, presente em duas amostras com 14 dias de maturação. Este fungo é encontrado em diversos alimentos, já tendo sido descrito em queijos, assim como a $B$. nivea, espécie encontrada em amostras de três períodos de maturação. Ambas espécies não causam danos ao alimento, porém a segunda pode produzir metabólitos tóxicos para o consumo, como a patulina, ácido bissoclâmico e ácido micofenólico, que são substâncias mutagênicas e neurotóxicas, potencialmente carcinogênica e imunossupressora, respectivamente (PITT \& HOCKING 2009).

O gênero Fusarium e as espécies $C$. herbarum e M. hiemalis já foram relatados em queijos. No QAS, o primeiro foi isolado em quatro amostras, e por ser componente da microbiota dos solos, sua presença no alimento é um indício de contaminação (PITT \& HOCKING 2009). As outras duas espécies estavam presentes em duas amostras cada, sendo o $C$. herbarum um importante microrganismo em queijos maturados (HAYALOGLU \& KIRBAG 2007).

$O$ G. candidum, presente em seis amostras, contribui na maturação de queijos macios e semi-duros, além de auxiliar na construção de sabores (MARCELLINO \& BENSON 1992), assim como o gênero Penicillium spp., isolado em cinco amostras, que é normalmente encontrado nestes derivados lácteos. No entanto, as espécies encontradas, $P$. chrysogenum, $P$. commune e $P$. expansum, são potenciais produtores de micotoxinas, como roquefortine $C$, ácido ciclopiazônico, patulina e citrinina. $\mathrm{O} P$. commune ainda é o principal causador de deterioração em queijos. (KURE et al. 2004, HAYALOGLU \& KIRBAG 2007).

As espécies encontradas no QAS, juntamente aos gêneros Rhizopus, Alternaria e Scopulariopsis, são isolados comuns de queijos (ERDOGAN et al. 2003, KORUKLUOGLU et al. 2005). ITULAIN M et al. (1996) também isolaram os gêneros Aspergillus, Geotrichum, Mucor e Penicillium nos queijos Idiazabal e Roncal. Já os estudos de LAVOIE et al. (2012) encontraram no "Quebec specialty" os gêneros Aspergillus, Cladosporium, Fusarium e Mucor. A pesquisa de TANIWAKI \& VAN DENDER (1992) isolou Aspergillus, Cladosporium e Penicillium de queijos tipo Parmesão e Prato, enquanto HAYALOGLU \& KIRBAG (2007) isolaram os gêneros Aspergillus, Cladosporium, Geotrichum, Mucor e Penicillium no queijo Kuflu.

Em relação ao QAS, os fungos filamentosos não podem ser considerados como caracterizadores do produto, uma vez que não estão presentes com frequência nas amostras, sendo provavelmente derivadas 
de contaminação no decorrer da produção, seja pela matéria-prima, equipamentos ou sala de maturação.

Em relação à variação da contagem de bolores ao longo dos períodos de maturação (Tabela 2), as médias se apresentaram entre 0,0 e 2,34 log UFC $\mathrm{g}^{-1}$, sendo que apenas a contagem dos queijos com 35 dias de maturação se mostraram estatisticamente diferentes dos queijos com 14 e 21 dias de maturação, quando submetidos ao teste de Tukey a $5 \%$ de significância.

Tabela 2. Contagem de fungos filamentosos presentes no Queijo Artesanal Serrano ao longo dos períodos de maturação.

Table 2. Count of filamentous fungi present in Serrano Artisanal Cheese throughout ripening periods.

\begin{tabular}{|c|c|c|c|c|}
\hline \multirow[t]{2}{*}{ Propriedades } & \multicolumn{4}{|c|}{ Contagens de Bolores log UFC g ${ }^{-1}$} \\
\hline & 14 dias & 21 dias & 28 dias & 35 dias \\
\hline 1 & 0,0 & 0,0 & 3,3 & 0,0 \\
\hline 2 & 3,0 & 0,0 & 0,0 & 0,0 \\
\hline 3 & 3,7 & 0,0 & 4,6 & 0,0 \\
\hline 4 & 0,0 & 0,0 & 0,5 & 0,0 \\
\hline 5 & 0,0 & 0,0 & 0,0 & 0,0 \\
\hline 6 & 0,0 & 4,9 & 3,5 & 0,0 \\
\hline 7 & 0,0 & 4,0 & 0,0 & 0,0 \\
\hline 8 & 4,0 & 0,0 & 3,0 & 0,0 \\
\hline 9 & 3,0 & 0,0 & 0,0 & 0,0 \\
\hline 10 & 0,0 & 5,4 & 0,0 & 0,0 \\
\hline 11 & 3,4 & 4,8 & 3,0 & 0,0 \\
\hline 12 & 4,0 & 5,1 & 0,0 & 0,0 \\
\hline 13 & 5,9 & 0,0 & 0,0 & 0,0 \\
\hline 14 & 4,5 & 3,3 & 0,0 & 0,0 \\
\hline 15 & 0,0 & 4,0 & 0,0 & 0,0 \\
\hline 16 & 0,0 & 3,0 & 3,8 & 0,0 \\
\hline 17 & 0,0 & 4,3 & 0,0 & 0,0 \\
\hline 18 & 0,0 & 2,6 & 0,0 & 0,0 \\
\hline 19 & 5,2 & 5,4 & 0,0 & 0,0 \\
\hline 20 & 0,0 & 0,0 & 4,1 & 0,0 \\
\hline Média & $1,83^{a}$ & $2,34^{a}$ & $1,29^{a, b}$ & $0,0^{b}$ \\
\hline Mínimo & 0,0 & 0,0 & 0,0 & 0,0 \\
\hline Máximo & 5,94 & 5,40 & 4,60 & 0,0 \\
\hline DP & 2,17 & 2,29 & 1,79 & - \\
\hline DPR (\%) & 118,67 & 97,62 & 138,52 & - \\
\hline
\end{tabular}

$\overline{\mathrm{DP}}=$ Desvio padrão, DPR $=$ Desvio padrão relativo. Médias seguidas de letras distintas diferem significativamente pelo teste de Tukey $(p=0,05)$.

As leveduras por sua vez apresentaram mais relevância do que os bolores, além de terem importância no processo de maturação deste produto (BERESFORD et al. 2001). As contagens das unidades formadoras de colônias estão apresentadas na tabela 3. Apenas a contagem de leveduras de 21 dias de maturação foi estatisticamente menor do que a contagem aos 28 dias de maturação, com média de 3,07 log UFC g-1 enquanto nos demais períodos a média variou entre 4,28 e 4,44 log UFC g ${ }^{-1}$.

Neste estudo, foram isoladas 82 culturas diferentes em 72 das 80 amostras, das quais apenas duas amostras com 14 dias de maturação e seis com 21 dias de maturação, não apresentaram crescimento (Tabela 4).

Os isolados são pertencentes a quatro gêneros: Candida, Kluyveromyces, Torulaspora e Trichosporon, com predomínio dos dois primeiros ao longo dos períodos de maturação.

A espécie isolada com maior frequência foi a $\mathrm{K}$. lactis, levedura que hidrolisa a lactose, produzindo outros açúcares que podem ser fonte de energia para outras espécies. A $K$. lactis também tem sido utilizada industrialmente como fonte de quimosina recombinante bovina para fabricação de queijos (JOHNSON \& ECHAVARRI-ERASUN 2011). Essa levedura também foi isolada com predominância sobre outras espécies em um estudo realizado com queijo Canastra produzido em Minas Gerais (NOBREGA 2007), e também no queijo Salitre, juntamente com a Debaryomyces hansenii, em estudo de LIMA et al. (2009), proveniente do 
mesmo estado. Já na pesquisa com o queijo Serro, a K. lactis foi detectada após 30 dias de maturação, indicando que, no produto estudado, a sua importância é maior em estágios mais avançados de maturação (CARDOSO et al. 2015), fato que se repetiu no QAS, porém com intervalos de tempo mais reduzidos, nos quais aos 14 dias houve o isolamento de três colônias, enquanto nos períodos seguintes foram entre sete e oito isolados, respectivamente.

Tabela 3. Contagem de leveduras presentes no Queijo Artesanal Serrano ao longo dos períodos de maturação.

Table 3. Yeast count in Serrano Artisanal Cheese throughout the ripening periods.

\begin{tabular}{ccccc}
\hline Propriedades & \multicolumn{4}{c}{ Contagens de Leveduras log UFC g $^{-1}$} \\
\hline 1 & 14 dias & 21 dias & 28 dias & 35 dias \\
\hline 2 & 4,0 & 1,8 & 4,3 & 5,2 \\
3 & 4,8 & 2,7 & 3,3 & 5,6 \\
4 & 4,2 & 3,9 & 5,8 & 5,2 \\
5 & 5,2 & 3,7 & 2,7 & 3,0 \\
6 & 3,1 & 4,7 & 2,7 & 4,6 \\
7 & 5,4 & 0,0 & 4,9 & 3,1 \\
8 & 5,8 & 5,9 & 5,7 & 4,5 \\
9 & 5,1 & 4,9 & 5,1 & 4,3 \\
10 & 4,5 & 4,2 & 4,3 & 3,7 \\
11 & 3,9 & 0,0 & 5,0 & 4,9 \\
12 & 4,9 & 0,0 & 4,7 & 4,3 \\
13 & 0,0 & 5,2 & 4,5 & 3,8 \\
14 & 5,3 & 3,8 & 4,0 & 4,8 \\
15 & 5,0 & 4,6 & 4,1 & 5,0 \\
16 & 5,5 & 5,7 & 5,6 & 4,3 \\
17 & 4,7 & 4,8 & 5,7 & 2,3 \\
18 & 5,0 & 0,0 & 4,0 & 4,9 \\
19 & 4,3 & 0,0 & 2,8 & 5,4 \\
20 & 0,0 & 0,0 & 4,4 & $4,28^{\mathrm{a}, \mathrm{b}}$ \\
Média & 5,7 & 5,4 & 4,9 & 2,32 \\
Mínimo & $4,32^{\mathrm{a}, \mathrm{b}}$ & $3,07^{\mathrm{a}}$ & 4,62 \\
Máximo & 0,0 & 0,0 & 2,73 & 0,91 \\
DP & 5,81 & 5,86 & 5,85 & 21,33 \\
DPR (\%) & 1,62 & 2,26 & 22,98 & \\
\hline
\end{tabular}

$\overline{\mathrm{DP}}=$ Desvio padrão, $\mathrm{DPR}=$ Desvio padrão relativo. Médias seguidas de letras distintas diferem significativamente pelo teste de Tukey $(p=0,05)$.

Outra levedura prevalente, foi a $C$. famata, em especial aos 14 dias de maturação. Este microrganismo é a fase anamorfa da D.hansenii. Esta espécie possui muita resistência ao sal, o que favorece sua condição de ser comumente encontrada em queijos (PITT \& HOCKING 2009), constituindo um agente importante no processo de maturação (ROOSTITA \& FLEET 1996). A mesma forma foi identificada no queijo Canastra, porém em menor frequência, apenas 16 das 118 amostras estudadas (NOBREGA 2007). Já a forma teleomorfa, D. hansenii, foi um isolado importante no queijo Salitre (LIMA et al. 2009), e também no queijo Serro, em diversos períodos de maturação (CARDOSO et al. 2015). Estudos recentes apontam que a $D$. hansenii pode ser usada como parte de culturas iniciadoras para fabricação de queijo, com a finalidade de desenvolvimento do sabor durante a maturação (SØRENSEN et al. 2011), corroborando os dados obtidos com o QAS.

A C. catenulata, isolada em 14 amostras, é uma espécie já reconhecida por estudos como uma das mais prevalentes em queijos artesanais, não só no Brasil, como também na Itália (PIRISI et al. 2011) e Dinamarca (SØRENSEN et al. 2011). Esta levedura já foi o isolado de maior prevalência no queijo Canastra por BORELLI et al. (2006), e juntamente com o K. lactis e o D. hansenii no queijo Salitre (LIMA et al. 2009). No queijo Serro também houve sua detecção, durante três períodos de maturação $(15,30$ e 60 dias) (CARDOSO et al. 2015). 
Ainda pode-se destacar a $C$. zeylanoides, que diferentemente de estudos com outros queijos artesanais brasileiros, apresentou frequência constante ao longo dos períodos de maturação, sendo isolada entre 10 e 15\% das amostras. No estudo realizado por NOBREGA (2007), também foi detectada esta espécie de levedura no queijo Canastra, porém em menor frequência, com duas amostras positivas das 118 analisadas.

Tabela 4. Espécies de leveduras presentes no Queijo Artesanal Serrano ao longo dos períodos de maturação identificadas por MALDI-TOF.

Table 4. Yeast species present in Serrano Artisanal Cheese during ripening periods identified by MALDITOF.

\begin{tabular}{lllllc}
\hline \multirow{2}{*}{ Gêneros } & \multicolumn{2}{c}{ Espécies } & \multicolumn{3}{c}{ Frequência de amostras com a presença de leveduras } \\
\cline { 2 - 6 } & & 14 dias $^{*}$ & 21 dias $^{*}$ & 28 dias $^{*}$ & 35 dias $^{*}$ \\
\cline { 2 - 6 } Candida & catenulata & $1(5 \%)$ & $3(15 \%)$ & $6(30 \%)$ & $4(20 \%)$ \\
& famata & $11(55 \%)$ & - & $6(30 \%)$ & $5(25 \%)$ \\
& intermedia & $1(5 \%)$ & - & $1(5 \%)$ & $1(5 \%)$ \\
& kefyr & - & $1(5 \%)$ & - & - \\
& krusei & - & & $1(5 \%)$ & $1(5 \%)$ \\
& pararugosa & - & $1(5 \%)$ & - & - \\
\hline Kluyveromyces & zeylanoides & $2(10 \%)$ & $2(10 \%)$ & $3(15 \%)$ & $3(15 \%)$ \\
\hline Torulaspora & lactis & $3(15 \%)$ & $8(40 \%)$ & $8(40 \%)$ & $7(35 \%)$ \\
\hline Trichosporon & delbrueckii & $1(5 \%)$ & $1(5 \%)$ & - & - \\
\hline Sem crescimento & ovoide & - & $1(5 \%)$ & - & - \\
\hline${ }^{*} \mathrm{n}=20$ & & $2(10 \%)$ & $6(30 \%)$ & $0(0 \%)$ & $0(0 \%)$ \\
\hline
\end{tabular}

As outras espécies de Candida (C. intermedia, C. kruser, C. kefyr e C. pararugosa) e o Trichosporon ovoide, foram apenas esporádicos durante a maturação do QAS, além de não estarem significativamente presentes em outros queijos artesanais do Brasil. Além disso, o T. ovoide não é um achado comum em alimentos, sendo um gênero componente da microbiota da pele humana (SILVESTRE JUNIOR et al. 2010), o que vai ao encontro do fato de ter sido isolado em apenas uma amostra. Por fim, a espécie $T$. delbrueckii foi encontrada em apenas duas amostras do QAS, em contrapartida, foi detectada em quase todos os períodos de maturação do queijo Serro (CARDOSO et al. 2015) e com alta predominância no queijo Canastra (NOBREGA 2007).

Neste estudo, o QAS produzido em Santa Catarina não se mostrou caracterizado pela presença de bolores. Os mesmos aparecem com pouca frequência e devido a contaminações no processo ou da micobiota do leite com o qual foi produzido. No entanto, a caracterização deste queijo pode ser dada pelos gêneros de leveduras, as quais prevalecem a $K$. lactis e algumas espécies do gênero Candida spp que se mostraram semelhantes a outros queijos artesanais produzidos no Brasil, porém com frequências de isolamento diferentes. Além disso, a variação da contagem de microrganismos se apresentou significativamente menor aos 21 dias de maturação em relação aos 28 dias para as leveduras, e aos 35 dias em relação aos 14 e 21 dias de maturação para os bolores. Dessa forma, o período de maturação não se mostrou atuante na contagem total desses microrganismos.

O QAS ainda necessita de mais estudos para definir seu padrão, principalmente com diferenciações do clima da região de produção, visto o investimento para reconhecimento da identidade geográfica do mesmo.

\section{AGRADECIMENTO}

À Coordenação de Aperfeiçoamento de Pessoal de Nível Superior (CAPES), pelo apoio financeiro e à Empresa de Pesquisa Agropecuária e Extensão Rural de Santa Catarina (EPAGRI) pela concessão das amostras.

\section{REFERÊNCIAS}

BERESFORD TP et al. 2001. Recent advances in cheese microbiology. International Dairy Journal 11: 259-274.

BORELLI BM et al. 2006. Yeast populations associated with the artisanal cheese produced in the region of Serra da Canastra, Brazil. World Journal of Microbiology and Biotechnology 22: 1115-1119. 
CARDOSO VM et al. 2015. The influence of seasons and ripening time on yeast communities of a traditional Brazilian cheese. Food Research International 69: 331-340.

CÓRDOVA UA et al. 2010. Queijo artesanal serrano: séculos de travessia de mares, serras e vales: a história nos campos da Serra Catarinense. Florianópolis: Epagri. 43p. (Documentos 234).

CÓRDOVA UA et al. 2011. O queijo artesanal serrano nos campos do Planalto das Araucárias Catarinense. Florianópolis: Epagri. 124p.

ERDOGAN A et al. 2003. Isolation of moulds capable of producing mycotoxins from blue mouldy Tulum cheeses produced in Turkey. International Journal of Food Microbiology 85: 83-85.

HAYALOGLU AA \& KIRBAG S. 2007. Microbial quality and presence of moulds in Kuflu cheese. International Journal of Food Microbiology 115: 376-380.

ITULAIN M et al. 1996. Estudio de los quesos com denominacion de origen Roncal e Idiazabal elaborados en Navarra. Alimentaria: Revista de tecnología e higiene de los alimentos 274: 69-72.

JAY JM. 2005. Microbiologia de Alimentos. 6.ed. Porto Alegre: Artmed. 712p.

JODRAL M et al. 1993. Mycoflora and toxigenic Aspergillus flavus in Spanish milk. International Journal of Food Microbiology 18: 171-174.

JOHNSON EA \& ECHAVARRI-ERASUN C. 2011. Yeast Biotechnology. In: BOEKHOUT CPKWF (Ed). The Yeasts. 5.ed. Londres: Elsevier. p. 21-44.

KORUKLUOGLU M et al. 2005. Mycoflora of some cheese samples in Bursa, Turkey. Indian Veterinary Journal 82: $340-$ 341.

KURE CF et al. 2004. Mould contamination in production of semi-hard cheese. International Journal of Food Microbiology 93: 41-49.

LAVOIE $\mathrm{K}$ et al. 2012. Characterization of the fungal microflora in raw milk and specialty cheeses of the province of Quebec. Dairy Science \& Tecnology 92: 455-468.

LIMA CDLC et al. 2009. Bactérias do ácido láctico e leveduras associadas com o queijo-de-minas artesanal produzido na região da Serra do Salitre, Minas Gerais. Arquivo Brasileiro de Medicina Veterinária e Zootecnia 61: 266-272.

MARCELLINO N \& BENSON DR. 1992. Scanning electron and light microscopic study of microbial succession on Bethlehem St. Nectaire cheese. Applied Environmental Microbiology 58: 3448-3454.

NAGY E et al. 2009. Species identification of clinical isolates of Bacteroides by matrixassisted laser-desorption/ionization time-of-flight mass spectrometry. Clinical Microbiology and Infection 15: 796-802.

NOBREGA JE. 2007. Caracterização do fermento endógeno utilizado na fabricação do queijo Canastra no município de Medeiros, Minas Gerais, com ênfase em leveduras. Dissertação (Mestrado em Ciência e Tecnologia de Alimentos). Viçosa: UFV. 82p.

PEREIRA BP et al. 2014. Implicações do processo produtivo na qualidade do Queijo Artesanal Serrano. Revista Eletrônica em Gestão, Educação e Tecnologia Ambiental 18: 116-126.

PIRISI A et al. 2011. Sheep's and goat's dairy products in Italy: Technological, chemical, microbiological, and sensory aspects. Small Ruminant Research 101: 102-112.

PITT JL \& HOCKING AD. 2009. Fungi and food spoilage. 3.ed. Londres: Blackie Academic \& Professional. 593p.

ROOSTITA R \& FLEET GH. 1996. The occurrence and growth of yeasts in Camembert and Blue-veined cheeses. International Journal of Food Microbiology 28: 393-404.

SANTA CATARINA. 2016. Lei $n^{\circ} 17003$, de 1 de setembro de 2016. Dispõe sobre a produção e comercialização do Queijo Artesanal Serrano, no Estado de Santa Catarina.

SILVA N et al. 2010. Manual de métodos de análise microbiológica de alimento e água. São Paulo: Varela. 535p.

SILVESTRE JUNIOR AM et al. 2010. Trichosporon species isolated from the perigenital region, urine and catheters of a Brazilian population. Brazilian Journal of Microbiology 41: 628-634.

SØRENSEN LM et al. 2011. Flavour compound production by Yarrowia lipolytica, Saccharomyces cerevisiae and Debaryomyces hansenii in a cheese-surface model. International Dairy Journal 21: 970-978.

TANIWAKI MH \& VAN DENDER AGF. 1992. Occurrence of Toxigenic Molds in Brazilian Cheese. Journal of Food Protection 55: 187-191. 